\title{
The Evaluation of Influence in Society's Information Networks
}

\author{
Ruiwei Feng \\ Department of Computing, North China Electricity Power University, Baoding 071000, China. \\ 736586335@qq.com
}

Keywords: Information Networks; Infectious disease model; Complex network; Topology; Control variable.

\begin{abstract}
In recent years, with the popularity and development of the Internet, information is spread more and more quickly. In this paper, based on a large amount of data, we first consider "Information transfer capability parameters of the information network" as an evaluation criterion to describe the information transfer capability of information network, and by virtue of the change rules to establish the prediction mechanism for this parameter. Then we introduce the expansion mode, which consider "the capability to accept information of the not disseminate nodes "as the evaluation criterion and describe the situations that public interest and opinion being changed through information networks. Ultimately, the model totally describe the conditions that the information spread among the information networks and nodes. Based on the model of infectious disease, the new model of this task is conducted. And then by the description of the information transfer capability of information network in different period, the model can predict the capability of transmitting information. It greatly simulate the topologies social network that exists today and describe the process of a complex network of information dissemination well.
\end{abstract}

\section{Introduction}

Great changes have been taking place for the development of information technology and information networks. Human beings are affected by various of news and make great use of these information to make decisions and even change the world. However, we found that there is no model that can be used to analyze the development of the information networks or people's reaction to the information.

According to the task about mass medium, we first searched for a lot of information about the transmission of the news and the development of the mass media then we found the model called SIR model which can help us in some ways.

\section{Method and Results}

We first analyze the task and introduce the dynamic model of differential equation, which is called SI Epidemic Model.[1]Based on the model introduced, we first find the identical point of the five period we consult. And each stage is the period of origin or prosperity of the newly-presented mass medium. The spread of news is on the basis of such systems: spreading between nodes whose function is accepting information directly, and spreading through mass medium. So we define the definition to represent the capability of communication, which is the average numbers of people who can contact the news through the two ways above.

We give the node two properties that are the probability of a person to accept a piece of message and the credibility of the news itself.[2] As time goes by, the process of communication can be imitated by the SI model. Knowing the rate of persons who know the news initially and the rate at a very moment, we can work out the daily dissemination capability of different period mentioned above by fitting at least squares principle. After that, we extend our model with the help of graph theory[3] and analyze the social network. In this period, we carry on numerical simulation to the existing social network. Through the simulation curve, capture real data through the whole process of information 
transmission in complex networks. Then further discuss the expansion of the model, we get the solution of how to accelerate information transmission, and guide the direction of public opinion.

\subsection{Terms, Definitions and Symbols}

i The percentage of people that the mass medium has covered from the total

$i_{0}$ The initial value of the statistics, that is the earliest-gained statistics

$t$ The interval of time between $i$ and $i(0)$

$\mathrm{p}$ The capability of receiving and non-spreading information of the node

$\lambda$ The capability of disseminating information of a network

\subsection{Assumptions}

Every adjacent years, $\lambda$ just change little. That is to say, it doesn't influence a lot from the news generation till the moment it committed successfully for the fast process of the information exchange.

During the period that we research, there is no force majeure coming across when the information spreading, which means the environment of our research is ideal.

\subsection{The Model}

\subsubsection{Basic Model}

When define the total number of people in the area, neither their birth or death, nor their immigration or emigration is considered.[4] People are divided into two groups and one of them called Susceptible while another called Infective. Besides, we define the percentage of these two kind of people in different moment as $s(t)$ and $i(t)$.

Assuming that if a patient contact with a healthy person, the healthy one could be infected. Each patients can get the amount of $\lambda s(t)$ persons infection. According to the datum, the percentage of patients in the total people is :

$$
i_{t}=\frac{1}{1+\left(\frac{1}{i_{0}}-1\right) \times e^{-\lambda t}}
$$

$\mathrm{i}_{0}$ : The initial percentage $\mathrm{t}$ : Times/day

\subsubsection{SI Model}

The spread of news from one to another can be regarded as an application of SI model. And it can be considered as a half discontinuity variation. Though looking through the past few decades or even look up to the future centuries, variation happens anytime. The selected period in the task is just several special points in the long history. However, the discontinuity can be more apparent if taking the situation of the recent period into consideration. Based on our practical problem, we correct the model appropriately and put forward our Model I as follows.[5]

$$
i_{t}=\frac{1}{1+\left(\frac{1}{i_{0}}-1\right) \times e^{-\lambda p t}}
$$

We define $\mathrm{P}_{2}$ representing the value of information, $\mathrm{P}_{1}$ representing people's initial opinion and bias, and $\mathrm{P}=\mathrm{P}_{1} \times \mathrm{P}_{2}$ as a variable to describe the degree of possibility to deliver the message successfully. Analyzing the several period and its time of starting and ending, as well as its ability to disseminate information, we predict the trend of curve. Based on the analyzed result, we can ultimately get to know which period is it now and the ability to disseminate information. The number of the period is defined as parameter $n$. After that, we predict the related data in the future 2050 and get the information dissemination capability.

Besides, we expand the former model to the SEIR model and continue to research network topology relation.

\section{Discussion}

In order to analyze the factors mentioned in the dissemination of information and how it influence public opinion, we take control of variables.

(1) Information Value 
The value of the variable control information $\left(V_{l}\right)$ were taken $0.25,0.35,0.45,0.55$ and the other variables still get the original value. We can get the curve of the percentage of node density as time goes by. [6] It can be seen from Figure. 1 that the higher the value of information, the faster it spread.

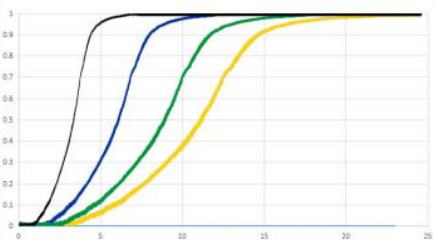

Figure. 1 : The density of Nodes(percentage)-Time(hour) curves

Yellow-0.25 Green-0.30

Blue-0.35 Black-0.40

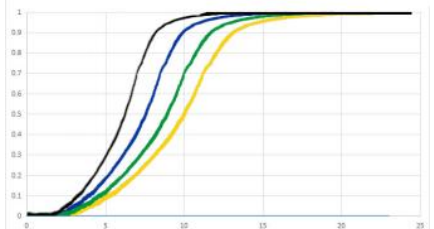

Figure. 2 : The density of

Nodes(percentage)-Time(hour) curves

Yellow-0.30 Green-0.35

Blue- 0.40 Black- 0.45

(2) People's First Prejudices

The value of the people first prejudices $\left(V_{2}\right)$ were taken $0.30,0.35,0.40,0.45$ and the other variables still get the original value. We can get the curve in the same way with the last one. As can be seen from Figure. 2, the greater the value of $V_{2}$, the greater the attractiveness of people(whether due to hatred or love). The result seems that it should be easier to spread.

(3) Message Form or Source

The value of the message form or source $i_{(0)}$ were taken $1,4,20,50$ and others are the same as the previous operation.

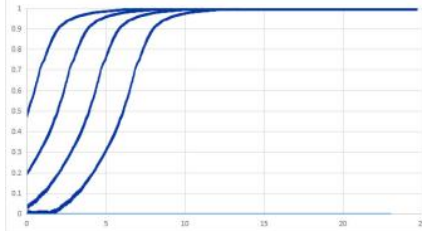

Figure. 3 : The density of Nodes(percentage)-Time(hour) curves

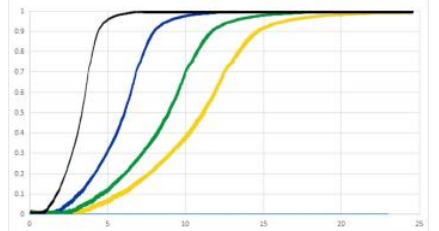

Figure. 4: The density of

Nodes(percentage)-Time(hour) curves

Yellow-20 Green-30 Blue-40 Black-300

As can be seen from Figure. 3, the initial status of $\mathrm{i}_{(0)}$ is determined and message form becomes more and more. Besides, the value of the scattering source is greater. But to the end, it achieve the same height.

\section{Conclusion}

Develop one or more model(s) that allow(s) people to explore the flow of information,then filter and find what is in line with the news. Then we take the Epidemic Model SI as our basic model and SEIR as our developed model. According to the assumption, we never consider message as news if the value of ' $p$ ' of this message can not satisfy the following conditions:

Table 1 : Evaluation standard of news[7, 8, 9]

\begin{tabular}{|c|c|c|c|c|}
\hline $\mathrm{P}$ & $<0.653$ & $0.653-0.826$ & $0.826-0.937$ & $0.937-1$ \\
\hline $\begin{array}{c}\text { News or Not } \\
\text { News }\end{array}$ & Not & Low-grade & High-grade & Higher-grade \\
News & News & News & News \\
\hline
\end{tabular}

Then we validate the model's reliability by using data in the past, predict the information communication situation today and compare that with the reality. We get a value of $\lambda$ in $2010 \mathrm{~s}$ as 0.7050 , with a elative error value of 0.0231 . Thus it can be seen that the model can predict well. Using the model to predict the communication networks' relationships and capacities in 2050. According to the model, it is the third period of revolution after the 2010s when in 2050. And the former two revolution would be in 2016 and 2040. In 2050, the value of $\lambda$ is 0.8823 and its capability of information dissemination has increased by $28.04 \%$ compared to that in the 2010 s. Rising from 0.689 
in the 2010 s to 0.8823 in 2050 , the data obviously suggest that the ability of information dissemination have a flying increase.

In the 1870s, 1920s, 1970s, 1990s and the 2010s, the major leader of the information network are separately newspaper, radio, television, personal computer and mobile phone. Before the coming revolution in 2050, the first period of revolution is just around the corner, which is the intelligent equipment represented by Apple Watch and Google Project Glass. It occurred to the public and accompany the human beings. Thus it can be seen that the mass medium is just facing for the direction that being smaller and more convenient. As the information network aid to be more vanguard in terms of technology, what it will be in the coming 2050 depends on the development of technology a lot .

\section{References}

[1] Yang Xuying. Based on SIR social network information dissemination model [D]. Xi'an University of Electronic Science and Technology, 2014.

[2] Wang Chao, Yang Xuying, Xu Ke, et al. SIR social network information dissemination model [J]. Chinese Journal of Electronics, 2014(11):2325-2330.

[3]XianzhiTechnologyCo.,Ltd.Xi'an. Theoretical Analysis of SEIR Spreading Model on P2P Specific Information[J]. Journal of xi 'an jiaotonguniversity, 2012, 46(2):31-37.

[4] BaiF.Infectious Disease Model[J]. Jornal of Ayan Normal Nvry Naral NDon, 2012

[5] The statistical yearbook of the United States.2002 2014.

[6] Li, J. Q., Zhang, J., \& Ma, Z. E. (2004). Global analysis of some epidemic models with general contact rate and constant immigration. Applied Mathematics \& Mechanics, 25(4), 396-404.

[7]Newspaper_Circulation_Trends_050611.pdf

[8] http://news.bbc.co.uk/2/hi/technology/8552410.stm

[9]The History and Present Condition of the Newspaper and Periodical Press of the United States.(S. N.D North,New York,1884. 